\title{
Ground-Based Observations of Terrestrial Gamma Ray Flashes Associated with Downward-Directed Lightning Leaders
}

\author{
Rasha Abbasi ${ }^{1, *}$, John belz ${ }^{1}$, Ryan Le Von ${ }^{1}$, Dan Rodeheffer $^{2}$, Paul Krehbiel ${ }^{2}$, Jackson Remington ${ }^{1}$, William Rison ${ }^{2}$, and \\ for the Telescope Array and TA/LMA Collaborations \\ ${ }^{1}$ Department of Physics, University of Utah, Salt Lake City, Utah, 84112, U.S.A. \\ ${ }^{2}$ Langmuir Laboratory for Atmospheric Research, New Mexico Institute of Mining and Technology, Socorro, New Mexico 87801, \\ U.S.A.
}

\begin{abstract}
Terrestrial gamma-ray flashes (TGFs) are bursts of gamma-rays initiated in the Earth's atmosphere. TGFs were serendipitously first observed over twenty years ago by the BATSE gamma ray satellite experiment. Since then, several satellite experiments have shown that TGFs are produced in the upward negative breakdown stage at the start of intracloud lightning discharges. In this proceeding, we present ground-based observation of TGFs produced by downward negative breakdown occurring at the beginning of negative cloud-to-ground flashes.

The Terrestrial gamma-ray flashes discussed in this work were detected between 2014-2017 at ground level by the Telescope Array surface detector (TASD) together with Lightning Mapping Array (LMA) and the slow electric field antenna (SA). The TASD detector is a $700 \mathrm{~km}^{2}$ ultra high energy cosmic ray detector in the southwestern desert of Utah. It is comprised of $507\left(3 \mathrm{~m}^{2}\right)$ plastic scintillator detectors on a $1.2 \mathrm{~km}$ square grid. The LMA detector, a three-dimensional total lightning location system, is comprised of nine stations located within and around the array. The slow electric field antenna records the electric field change in lightning discharges.

The observed Gamma ray showers were detected in the first 1-2 ms of downward negative breakdown prior to cloud-to-ground lightning strikes. The shower sources were observed by the LMA detector at altitudes of a few kilometers above ground level. The detected energetic burst showers have a footprint on the ground typically $3-5 \mathrm{~km}$ in diameter. The bursts comprise of several (2-5) individual pulses, each of which have a span of a few to tens of microseconds and an overall duration of several hundred microseconds. Using a forward-beamed cone of half-angle of 16 degrees, GEANT simulation studies indicate that the showers are consistent with gamma rays of $10^{12}-10^{14}$ primary photons. We hypothesize that the observed terrestrial gamma-ray flashes are similar to those detected by satellites, but that the ground-based observations are closer to the source and therefore are able to observe weaker sources and report on the structure of the temporal distribution at the source. This result and future studies will enable us to better identify and constrain the mechanisms of downward TGF production.
\end{abstract}

\section{Introduction}

Terrestrial gamma-ray flashes (TGFs) are bursts of gamma-rays initiated in the Earth's atmosphere. The first detection of TGFs was reported in 1994 by the Burst and Transient Source Experiment (BATSE) on the Compton Gamma-Ray Observatory satellite [1,2]. Since then, several observations have shown that satellite-detected TGFs are associated with lightning flashes. In a normal polarity thunderstorm, a high-level intra-cloud lightning flash begins with a negative leader propagating upward from the mid-level negative charge region towards the upper positive charge region [3-7]. If upward-propagating negative leaders produce TGFs beamed upward to satellite detectors, then a downward-propagating negative leaders might also produce TGFs which are beamed downward.

Several bursts were observed by the Telescope Array (TA), an Ultra High Energy Cosmic Rays (UHECRs) de-

\footnotetext{
*e-mail: rasha@cosmic.utah.edu
}

tector, between 2008 and 2013 [8]. The bursts triggered the detector with a rate much higher than that expected by an accidental coincidence of UHECRs. These bursts were found to correlate with local lightning activity. A Lightning Mapping Array (LMA) and Slow electric field Antenna (SA) were installed at the TA site in order to study the effect.

In this proceeding, we report on the first joint gammaray observations with the TA/LMA and Slow Antenna instruments. Each of the gamma-ray events detected during the downward initial negative breakdown at the beginning of several kilomenters AGL altitude intracloud flashes. In each case the parent flash was a (negative Cloud to Ground) -CG discharge and the burst occurred within the first or second millisecond of the flash. Such breakdown maybe the downward analog the TGF phenomenon observed by satellite borne experiments. 


\section{Lightning Detection at TA}

The TA detector is the largest UHECRs in the northern hemisphere. It is located in Millard County, Utah and is composed of 507 scintillator detectors on a $1.2 \mathrm{~km}$ square grid covering $700 \mathrm{~km}^{2}$ Surface Detector (SD) array [9]. The Telescope Array Surface Detector (TASD), has an approximately $100 \%$ duty cycle, and provides shower footprint information including core location, lateral density profile, and timing.

Each SD unit consists of upper and lower scintillator planes, each plane is $3 \mathrm{~m}^{2}$ in area, and is $1 \mathrm{~cm}$ thick. The upper and lower planes are separated by a $1 \mathrm{~mm}$ thick steel plate, and are read out by individual photomultiplier tubes (PMTs) which are coupled to the scintillator via an array of wavelength-shifting fibers. The scintillator, fibers and photomultipliers are contained in a light-tight and electrically grounded stainless steel box $(1.5 \mathrm{~mm}$ thick on top and $1.2 \mathrm{~mm}$ thick on the bottom) under an additional $1.2 \mathrm{~mm}$ iron roof providing protection from extreme temperature variations [9].

The TASD event trigger is recorded when three adjacent SDs observe a time-integrated signal greater than 3 Vertical Equivalent Muon (VEM) within $8 \mu \mathrm{s}$. When a trigger occurs, the signals from all SDs detecting an integrated amplitude greater than $0.3 \mathrm{VEM}$ within $\pm 32 \mu$ s are also recorded. The VEM is a unit of energy deposit, equivalent to the energy deposited in a single TASD scintillator plane by a vertical relativistic muon. In more conventional units a VEM is about $2 \mathrm{MeV}$ per scintillator plane, roughly 30 ADC counts above background.

In 2013 the Lightning Mapping Array (LMA) was deployed and running at the Telescope Array site. LMA was developed at the Langmuir Laboratory for Atmospheric Research [10,11]. LMA is an ideal instrument for studying electrical discharges in the Earth's atmosphere. Nine LMA detectors were deployed throughout the TA detector. The Langmuir LMA utilizes low-VHF (60-66 MHz) radio emissions in order to create 3-dimensional reconstruction of a lightning flash. It is most sensitive when deployed in radio-quiet, rural areas like the southwestern desert of the State of Utah. Each LMA detector records time and amplitude of impulses above trigger threshold. A fit is performed on the GPS-timed arrival of these impulses in order to determine the position and time of each unique LMA source. Six or more detectors are required to determine the location and time of the source events.

In 2014, a slow antenna [12] was also deployed at the Telescope Array site in order to measure changes in the ambient electric field. The slow antenna is located in the center of the TASD. It consists of a flat metal plate centered in a grounded metal bowl and records voltage proportional to the electric field at the surface of the plate. The slow antenna has a low-frequency time constant of 10 seconds, and an upper frequency response of $25 \mathrm{kHz}$. The nominal sensitivity of the sensors allows us to accurately measure field changes in the range of $10 \mathrm{mV} / \mathrm{m}$ to $10 \mathrm{kV} / \mathrm{m}$.

The TASD covers an area hundreds of times larger than other ground-based detectors of lightning-associated
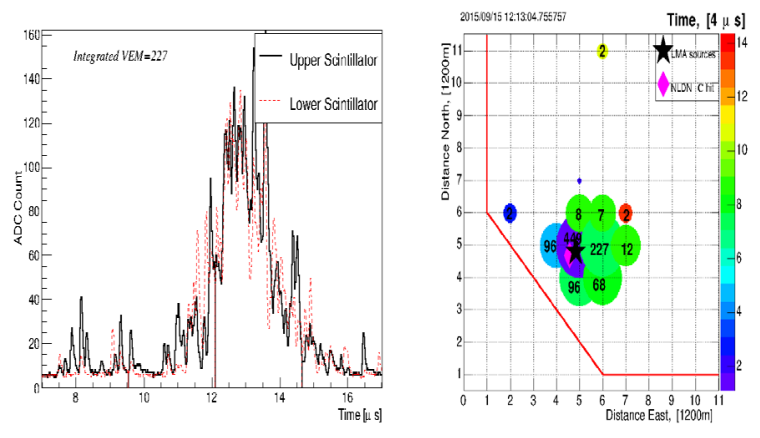

Figure 1. Left: Upper and lower scintillator waveforms in a single surface detector unit, for the second trigger in the LMAcorrelated TASD burst observed at 12:13:04 on 15 Sept. 2015. Right: Footprint of TASD hits for all detectors units involved in the second trigger of the burst, with the numbers indicating the Vertical Equivalent Muon (VEM) counts, and the color indicating the relative arrival times. Initial LMA and NLDN events are indicated by stars and diamonds respectively. The red line indicates the southwestern boundary of the TASD array.

events, making it the largest such detector to date. The addition of an LMA network and $\triangle \mathrm{E}$ observations to the TASD provides us with a unique suite of instruments for studying the TGF phenomena from the ground.

\section{Observations}

Following Okuda et. al. [8]. In this study we searched for candidate lightning events in the TASD dataset by identifying instances in which "bursts" of consecutive TASD triggers were recorded in $1 \mathrm{~ms}$ time intervals. Since the TASD mean trigger rate is less than $0.01 \mathrm{~Hz}$, it is extremely unlikely that such a burst could be caused by accidental coincidence of high-energy cosmic rays. We report here on fifteen such bursts.

A typical trigger burst event waveform recorded by an SD together with the corresponding TASD footprint $(\sim 3-$ $5 \mathrm{~km}$ in diameter) is shown in Figure 1. Each circle in the TASD footprint is a triggered SD with a color related to its relative arrival time. The size of the circle is proportional to the logarithm of the energy deposited in the SD. The number on each circle is the integrated area under the photomultiplier waveform VEM (one VEM corresponds to $\approx$ $2 \mathrm{MeV}$ ). The LMA and the the Vaisala National Lightning Detector Network (NLDN) source events are indicated by stars and diamonds respectively.

Typically the bursts occurred during the first $1-2 \mathrm{~ms}$ of the discharges and have overall durations between 87 and $551 \mu \mathrm{sec}$. With the high-resolution timing of the TASD, the bursts are found to consist of several (2-5) individual components as shown in Figure 2, each of which are a few microseconds in duration, separated in time by $\simeq 10$ $250 \mu \mathrm{s}$.

\subsection{LMA and SA-Correlated TASD Bursts}

From 2014-2016 six energetic radiation bursts were found to be in coincidence with LMA activity. The TASD trig- 


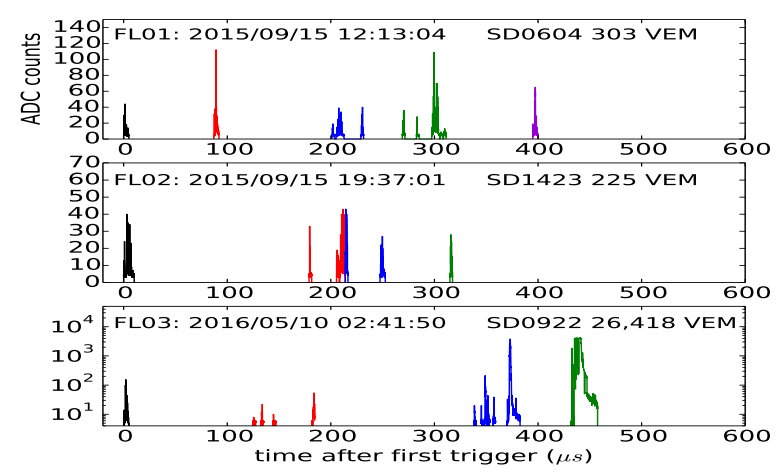

Figure 2. Combined waveforms, for one of the SDs, for all triggers observed in three selected bursts. Each trigger in the burst is colored individually. Triggers 1 through 5 are colored in black, red, blue, green and violet. The waveforms are found to be temporally resolved into discrete components, most of which are less than 10 microseconds in duration and which occur in succession over a duration of a few hundred microseconds.

gers occurred as the initial negative breakdown descended toward and into the lower positive charge region.

In most of the Flashes, the LMA observations show that the heights of the TASD trigger events can be estimated quantitatively by averaging the altitudes of the the LMA events leading up to the triggers. The average initial activity was a few kilometers above the TASD triggers. After the 1-2 ms of the first LMA source observation the TASD was triggered, the flashes spent 200 and $300 \mathrm{~ms}$ discharging the large regions of lower positive charge before producing negative strokes to ground.

Slow electric field change data was only collected during the 2014 storm season. Seven TASD trigger bursts were correlated with the Slow Antenna observations in 2014. These triggers was observed by the Slow Antenna alone, due to the intermittent coverage of the LMA detector in that period. The SA leader field changes observation durations ranged from short to fast durations. The TASD trigger occurred in the first ms of the flashes, and were consistent with downward negative leader breakdown that was followed by a return stroke peak current that ranged widely from $-11.7--140 \mathrm{kA}$.

Note that even though there is no direct altitude data for these SA-correlated flashes. The TASD footprints are comparable for the LMA and the SA events in both timing of the TASD observations relative to the beginning of the flash and the location of the flash relative to the TASD trigger (provided by NLDN).

This correlation between the SA/TASD and LMA/TASD observations was confirmed by an event observed on 12 September 2017 using the TASD, SA, LMA, and NLDN shown in Figure 3. The TASD trigger was observed one ms after and directly below the first LMA observed source. The SA field change observations shows the TASD trigger for this event is consistent with downward negative leader breakdown. The LMA observations show that the heights of the sources of the TASD trigger bursts is about $3.5 \mathrm{~km}$. It is worth noting that from the NLDN data, the storm starts with multiple

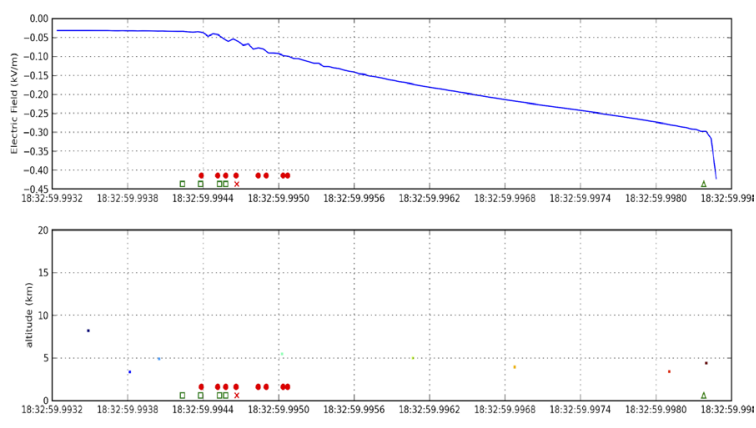

Figure 3. top: The change in the Electric Field $[\mathrm{kV} / \mathrm{m}]$ vs. time for the SA-correlated TASD burst observed at 18:32:00 on 12 Sept. 2017 in blue line while the TASD burst is shown in filled red circles. The NLDN observations are shown in green squared (IC) and triangles (CG). bottom: The altitude [km] vs. time as observed by LMA (in filled squares).

IC observations with a peak current of $-20 \mathrm{kA}$ followed by quite an energetic CG w/peak current of $-113 \mathrm{kA}$.

\section{Event Simulation}

The principal question is whether the observed showers are indeed created mainly by gamma-rays - usually defined as having energies greater than $100 \mathrm{keV}$ - or whether the predominant source of energy deposit in the TASD is due to lower-energy $x$-rays.

A simple argument can be made on the basis of the effects of attenuation and scattering in the atmosphere above the TASD. The absorption length of photons in the atmosphere plateaus above $100 \mathrm{keV}$ to about $100 \mathrm{~m}$ at TA altitudes [13]. Because the leaders associated with the TASD occur at up to $4 \mathrm{~km}$ above ground level and leave footprints on the ground of a few kilometers in size, we can expect that substantial attenuation of lower-energy photons will occur in the atmospheric above the detector.

In order to be more quantitative, we performed a GEANT4 simulation [14] incorporating a model of the atmosphere as well as the TASD [15]. We varied the energy and altitude of the primary photons, and recorded the total energy deposited in the TASD.

The results of this simulation are summarized in Figure 4, showing the mean TASD energy deposit versus altitude for various primary photon energies. The effect of decreasing photon attenuation in the $x$-ray to gammaray transition range is significant: At an altitude of $1 \mathrm{~km}$ AGL, the mean energy deposited by a $1 \mathrm{MeV}$ photon is a factor of five orders of magnitude greater than that of a $100 \mathrm{keV}$ photon. For reasonable energy spectra [16] the corresponding decrease in flux is far less, only one or two orders of magnitude. Thus from the GEANT4 simulation the TASD signal is due to primary photons with energy of order $1 \mathrm{MeV}$ or greater.

The sources number of primary photons were also estimated. The number of primary photons are highly dependent on the simulation variables such as the productions altitude and the openning angle of the cone. With the 
TASD we have the unique opportunity to use for the first time the full footprint size of the shower $(\sim 3-5 \mathrm{~km}$ in diameter) information in the simulation. This together with knowing the sources heights and the fact that the LMA and the NLDN sources were also found to be directly above the TASD bursts, we conclude that the observed showers are forward beamed within a cone of approximately half-angle $16^{\circ}$. The angular distribution of particles is assumed to be isotropic within that cone. Showers are simulated using GEANT4 simulation following the RREA spectrum $[17,18]$ and are then tracked through the atmosphere and the TASD detector model. The observations were found to be consistent with fluence of $\simeq 10^{12}-10^{14}$ photons.

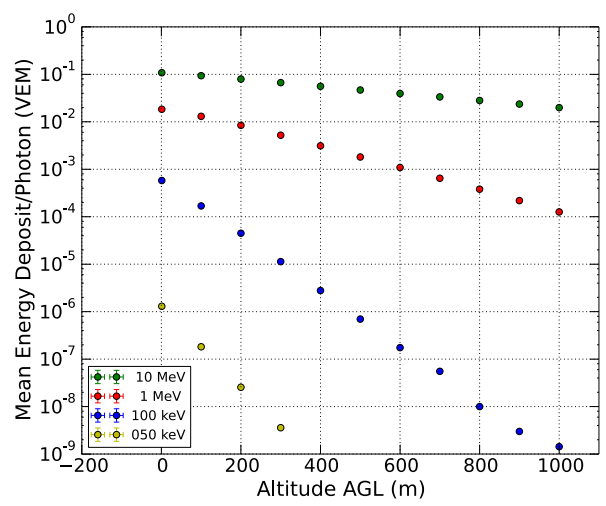

Figure 4. Mean energy deposit (average of two TASD planes) per primary photon, versus altitude AGL for various photon energies.

\section{Summary and Discussion}

Over a three-year period between 2014 and 2017, a total of fifteen TGF bursts were identified for which 3-D LMA and/or $\Delta \mathrm{E}$ lightning measurements were available. In each case:

- The parent flash was a -CG discharge and the burst occurred within the first or second millisecond of a downward negative leader activity.

- The burst durations were found to be within several hundred microseconds, with sub showers of few to few tens of microseconds.

- The sources altitude were typically of a few kms or less above ground level.

- The showers were forward beamed with footprints of 3$5 \mathrm{kms}$ in diameter.

From simulation, we conclude that the observed TASD bursts are due to primary gamma-ray showers. Moreover, the observed energy deposit of the shower found to be consistent with fluence of $\simeq 10^{12}-10^{14}$ photons.

The observed burst-like nature of the gamma radiation seen in the present study is consistent with being produced by the stepping process of negative-polarity breakdown. This is the downward analog of the satellite-born observed TGFs found to be produced by upward negative breakdown at the beginning of intracloud discharges. It is worth noting that, the TASD bursts observations are in full agreement with the assessment by [19] that the longer duration $\geq 100 \mu$ s TGF pulses of satellite observations could readily be due to overlapping emissions (due to Compton Scattering) from much shorter $(\sim 10 \mu \mathrm{s})$ temporal durations.

Currently, the LMA network, three slow antennas, a fast antenna, and an Interferometer (INTF) are deployed at the Telescope Array site. Moreover, an expansion by a factor of four in the coverage area of TASD is planned within the next several years. This will enable us to study the relation between SD observations and the development of negative breakdown in greater detail.

\section{References}

[1] G. J. Fishman et al., Science 264, 1313 (1994).

[2] C. Kouveliotou, The Astrophysical Journal Supplement 92, 637 (1994).

[3] Stanley et al., Geophysical Research Letters 33 (2006), 106803.

[4] X. Shao et al., J. Geophys. Res. 115, A00E30 (2010).

[5] G. Lu et al., Geophys. Res. Lett. 37, L11806 (2010).

[6] S. A. Cummer et al., Geophys. Res. Lett. 42, 7792-7798 (2015).

[7] F. Lyu et al., Geophys. Res. Lett. 43 (2016).

[8] R. Abbasi et al., Physics Letters A 381, 2565 (2017).

[9] T. Abu-Zayyad et al. (Telescope Array), Nucl. Instrum. Meth. A689, 87 (2013), arXiv:1201.4964 [astro-ph.IM] .

[10] W. Rison et al., Geophysical Research Letters 26, 3573 (1999).

[11] R. J. Thomas, , et al., Journal of Geophysical Research: Atmospheres 109 (2004), d14207.

[12] P. R. Krehbiel et al., J. Geophys. Res. 84, 2432 (1979).

[13] C. Patrignani et al. (Particle Data Group), Chin. Phys. C40, 100001 (2016).

[14] S. Agostinelli et al. (GEANT4), Nucl. Instrum. Meth. A506, 250 (2003).

[15] D. Ivanov, Energy Spectrum Measured by the Telescope Array Surface Detector, Ph.D. thesis, University of Utah (2012).

[16] Dwyer et al., Journal of Geophysical Research: Space Physics 117 (2012), a10303.

[17] J. R. Dwyer and D. M. Smith, Geophysical Research Letters 32 (2005), 122804.

[18] J. R. Dwyer et al., Space Science Rev. 173, 133 (2012)

[19] Celestin, S., and Pasko, V.P. (2012), Compton scattering effects on the duration of terrestrial gammaray flashes, Geophys. Res. Lett. 39 L02802. 